\title{
Research Article \\ Probabilistic Quadratic Programming Problems with Some Fuzzy Parameters
}

\author{
S. K. Barik and M. P. Biswal \\ Department of Mathematics, Indian Institute of Technology, Kharagpur, Kharagpur 721 302, India \\ Correspondence should be addressed to M. P. Biswal, mpbiswal@maths.iitkgp.ernet.in
}

Received 11 October 2011; Revised 9 December 2011; Accepted 20 December 2011

Academic Editor: Shangyao Yan

Copyright (C) 2012 S. K. Barik and M. P. Biswal. This is an open access article distributed under the Creative Commons Attribution License, which permits unrestricted use, distribution, and reproduction in any medium, provided the original work is properly cited.

\begin{abstract}
We present a solution procedure for a quadratic programming problem with some probabilistic constraints where the model parameters are either triangular fuzzy number or trapezoidal fuzzy number. Randomness and fuzziness are present in some real-life situations, so it makes perfect sense to address decision making problem by using some specified random variables and fuzzy numbers. In the present paper, randomness is characterized by Weibull random variables and fuzziness is characterized by triangular and trapezoidal fuzzy number. A defuzzification method has been introduced for finding the crisp values of the fuzzy numbers using the proportional probability density function associated with the membership functions of these fuzzy numbers. An equivalent deterministic crisp model has been established in order to solve the proposed model. Finally, a numerical example is presented to illustrate the solution procedure.
\end{abstract}

\section{Introduction}

In real-world applications, it is very much difficult to know all the information about the input parameters of the mathematical programming model because relevant data are inexistent or scarce, difficult to obtain or to estimate, the system is subject to changes, and so forth, that is, input parameters are uncertain. One of the best ways of modeling these uncertainties in the form of mathematical programming is known as stochastic programming. It is widely used in several research areas such as agriculture, capacity planning, finance, forestry, military, production control and scheduling, sport, telecommunications, transportation, environmental management planning.

It should also be noted, that in many practical situations, knowledge about the data (i.e., the coefficients / parameters of the model) is not purely probabilistic or possibilistic but 
rather a mixture of both kinds. For an example consider a firm that desires to maximize its profit by meeting all the customers demands which fluctuate due to random change in price. By the stochasticity of the demand and the fact that the production may not fulfill all the possible demands, this point cannot be satisfactorily answered by the true or false statement. This optimization problem is related to two types of uncertainties, namely, randomness and fuzziness, which motivates the proposed study in this direction.

Quadratic programming $(\mathrm{QP})$ is an optimization technique where we minimize/maximize a quadratic objective function of several variables subject to a set of linear constraints. QP has a wide range of applications, such as portfolio selection, electrical energy production, agriculture, and crop selection. Probabilistic quadratic programming is applicable for financial and risk management. In this direction several important-related literatures are found, which are cited below.

McCarl et al. [1] presented some of the methodologies where QP is applicable. Liu and Wang [2] proposed an interval quadratic programming problem where the cost coefficients, constraint coefficients, and right-hand sides are represented by interval parameters. Silva et al. [3] developed an original and novel fuzzy sets based method that solves a class of quadratic programming problems with vagueness. Liu [4] presented a fuzzy quadratic programming problem where the cost coefficients, constraint coefficients, and right-hand sides values are represented by fuzzy data. Ammar [5] proposed a multiobjective quadratic programming problem having fuzzy random coefficient matrix in the objectives and constraints, where the decision vector is treated as a fuzzy vector. Liu [6] discussed the fuzzy quadratic programming problem where the cost coefficients, constraint coefficients, and right-hand side parameters of the constraints are represented by convex fuzzy numbers. He also described a solution procedure [7] for a class of fuzzy quadratic programming problems, where the cost coefficients of the objective function, constraint coefficients, and righthand side parameters values are fuzzy numbers. Qin and Huang [8] presented an inexact chance constrained quadratic programming model for stream water quality management. Nasseri [9] defined a quadratic programming problem with fuzzy numbers where the cost coefficients, constraint coefficients, and right-hand side parameters values are represented by trapezoidal and/or triangular fuzzy numbers. An inexact fuzzy-stochastic quadratic programming method is developed by Guo and Huang [10] for effectively allocating waste to a municipal solid waste management system by considering the nonlinear objective function and multiple uncertainties on parameters in the constraints.

In this paper, we develop a new method for solving probabilistic quadratic programming problems involving some of the coefficients that are triangular fuzzy numbers and/or trapezoidal fuzzy numbers. Only the right- hand side parameters of the constraints are considered as Weibull random variables with known probability distributions. Both randomness and fuzziness are considered together within the QP framework. A defuzzification method is introduced for finding the crisp values of the fuzzy numbers using the Mellin transformation [11].

\subsection{Probabilistic Fuzzy Quadratic Programming Problem}

A probabilistic fuzzy quadratic programming problem is a modified QP having a quadratic objective function and some linear constraints involving fuzziness and randomness in some situations. When some of the input parameters of the QP are characterized by stochastic and 
fuzzy parameters, the problem is treated as a probabilistic fuzzy quadratic programming problem. A general probabilistic fuzzy quadratic programming problem can be presented as

$$
\min : z=\sum_{j=1}^{n} \widetilde{c}_{j} x_{j}+\frac{1}{2} \sum_{i=1}^{n} \sum_{j=1}^{n} \tilde{q}_{i j} x_{i} x_{j}
$$

subject to

$$
\begin{gathered}
\operatorname{Pr}\left(\sum_{j=1}^{n} \tilde{a}_{i j} x_{j} \leq b_{i}\right) \geq 1-\alpha_{i}, \quad i=1,2, \ldots, m, \\
x_{j} \geq 0, \quad j=1,2, \ldots, n
\end{gathered}
$$

where $0<\alpha_{i}<1, i=1,2, \ldots, m$, are the specified probabilities. The coefficients $\tilde{c}_{j}, j=$ $1,2, \ldots, n$, and $\tilde{q}_{i j}, i=1,2, \ldots, n, j=1,2, \ldots, n$, are considered as triangular fuzzy numbers but $\tilde{a}_{i j}, i=1,2, \ldots, m, j=1,2, \ldots, n$, are consider as trapezoidal fuzzy number. Only $b_{i}$, $i=1,2, \ldots, m$, are considered as random variables with known distributions. The decision variables $x_{j}, j=1,2, \ldots, n$, are treated as deterministic in the problem. In the following section, we discuss some useful preliminaries related to fuzzy numbers, then the method of defuzzification is introduced.

\section{Some Preliminaries}

In this section, we present triangular and trapezoidal membership functions that are used in the model formulation. Also we introduce the Mellin transform to find the expected value of the function of a random variable using proportional probability density function associated with the membership functions of the fuzzy numbers.

Definition 2.1 (triangular fuzzy number). A fuzzy number denoted by the triplet $\tilde{A}=$ $\left(a_{1}, a_{2}, a_{3}\right)$ is called a triangular fuzzy number with a piecewise linear membership function $\mu_{\tilde{A}}(x)$ defined by

$$
\mu_{\widetilde{A}}(x)= \begin{cases}\frac{x-a_{1}}{a_{2}-a_{1}}, & a_{1} \leq x \leq a_{2} \\ \frac{a_{3}-x}{a_{3}-a_{2}}, & a_{2} \leq x \leq a_{3} \\ 0, & \text { otherwise. }\end{cases}
$$


Definition 2.2 (trapezoidal fuzzy number). A fuzzy number denoted by the quadruplet $\tilde{A}=\left(a_{1}, a_{2}, a_{3}, a_{4}\right)$ is called a trapezoidal fuzzy number with a piecewise linear membership function $\mu_{\tilde{A}}(x)$ defined by

$$
\mu_{\tilde{A}}(x)= \begin{cases}\frac{x-a_{1}}{a_{2}-a_{1}}, & a_{1} \leq x \leq a_{2}, \\ 1, & a_{2} \leq x \leq a_{3} \\ \frac{a_{4}-x}{a_{4}-a_{3}}, & a_{3} \leq x \leq a_{4}, \\ 0, & \text { otherwise. }\end{cases}
$$

\subsection{Defuzzification with Probability Density Function and Membership Function}

Let $F(\mathbb{R})$ be the set of all fuzzy numbers. Let $\left(a_{1}, a_{2}, a_{3}\right)$ and $\left(a_{1}, a_{2}, a_{3}, a_{4}\right)$ be the triangular and trapezoidal fuzzy numbers, respectively, in $F(\mathbb{R})$. Now we define the method associated with a probability density function for the membership function of $\widetilde{A}$ as follows $[12,13]$.

Proportional probability distribution: define a probability density function $f_{1}=c \mu_{\tilde{A}}(x)$ associated with $\widetilde{A}$, where $c$ is a constant obtained by using the property of probability density function, that is, $\int_{-\infty}^{\infty} f_{1}(x) d x=1$, that is, $\int_{-\infty}^{\infty} c \mu_{\tilde{A}}(x) d x=1$.

\subsection{Mellin Transform}

As we know that any probability density function with finite support is associated with an expected value, the Mellin transform $[12,13]$ is used to find this expected value.

Definition 2.3. The Mellin transform $M_{X}(t)$ of a probability density function $f(x)$, where $x$ is a positive, is defined as

$$
M_{X}(t)=\int_{0}^{\infty} x^{t-1} f(x) d x
$$

where the integral exists.

Now we find the Mellin transform in terms of expected values. Recall that the expected value of any function $g(X)$ of the random variable $X$, whose probability density function is $f(x)$, is given by

$$
\mathbb{E}[g(X)]=\int_{-\infty}^{\infty} g(x) f(x) d x
$$

Therefore, it follows that $M_{X}(t)=\mathbb{E}\left[X^{t-1}\right]=\int_{0}^{\infty} x^{t-1} f(x) d x$.

Hence, $\mathbb{E}\left[X^{t}\right]=M_{X}(t+1)$. Thus, the expected value of random variable $X$ is $\mathbb{E}[X]=$ $M_{X}(2)$.

For an example, if $\tilde{A}_{1}=\left(a_{1}, a_{2}, a_{3}\right)$ and $\tilde{A}_{2}=\left(a_{1}, a_{2}, a_{3}, a_{4}\right)$ are the triangular and trapezoidal fuzzy numbers, respectively, then their crisp values are calculated by finding 
expected values using the probability density function corresponding to the membership functions of the given fuzzy number.

Now, the probability density function corresponding to triangular fuzzy number $\widetilde{A}_{1}=$ $\left(a_{1}, a_{2}, a_{3}\right)$ is given as

$$
f_{\widetilde{A}_{1}}(x)=c_{1} \mu_{\widetilde{A}_{1}}(x)
$$

where $\mu_{\widetilde{A}_{1}}(x)$ is defined as

$$
\mu_{\tilde{A}_{1}}(x)= \begin{cases}\frac{x-a_{1}}{a_{2}-a_{1}}, & a_{1} \leq x \leq a_{2} \\ \frac{a_{3}-x}{a_{3}-a_{2}}, & a_{2} \leq x \leq a_{3} \\ 0, & \text { otherwise }\end{cases}
$$

Now $c_{1}$ is calculated as

$$
\int_{-\infty}^{\infty} f_{\widetilde{A}_{1}}(x) d x=1
$$

that is,

$$
\int_{-\infty}^{\infty} c_{1} \mu_{\widetilde{A}_{1}}(x) d x=1
$$

that is,

$$
c_{1} \int_{a_{1}}^{a_{2}} \frac{x-a_{1}}{a_{2}-a_{1}} d x+c_{1} \int_{a_{2}}^{a_{3}} \frac{a_{3}-x}{a_{3}-a_{2}} d x=1
$$

On integration, we get

$$
c_{1}=\frac{2}{a_{3}-a_{1}}
$$

The proportional probability density function corresponding to triangular fuzzy number $\widetilde{A}_{1}$ is given by

$$
f_{X_{\tilde{A}_{1}}}(x)= \begin{cases}\frac{2\left(x-a_{1}\right)}{\left(a_{2}-a_{1}\right)\left(a_{3}-a_{1}\right)}, & a_{1} \leq x \leq a_{2} \\ \frac{2\left(a_{3}-x\right)}{\left(a_{3}-a_{2}\right)\left(a_{3}-a_{1}\right)}, & a_{2} \leq x \leq a_{3} \\ 0, & \text { otherwise }\end{cases}
$$

Graphically it is shown in Figure 1. 


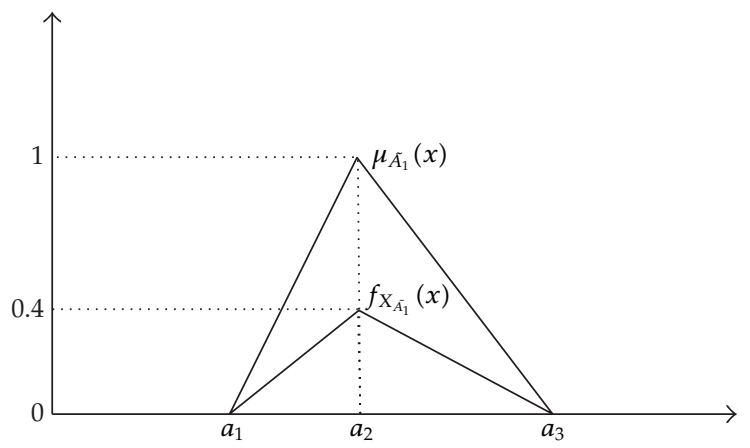

Figure 1: Proportional probability density function of triangular fuzzy number.

Further, using the Mellin transform, we obtain

$$
M_{X}(t)=\int_{0}^{\infty} x^{t-1} f_{\tilde{X}_{\tilde{A}_{1}}}(x) d x=\int_{a_{1}}^{a_{2}} x^{t-1} \frac{2\left(x-a_{1}\right)}{\left(a_{2}-a_{1}\right)\left(a_{3}-a_{1}\right)} d x+\int_{a_{2}}^{a_{3}} x^{t-1} \frac{2\left(a_{3}-x\right)}{\left(a_{3}-a_{2}\right)\left(a_{3}-a_{1}\right)} d x
$$

On integration, we obtain

$$
M_{\tilde{X}_{\tilde{A}_{1}}}(t)=\frac{2}{\left(a_{3}-a_{1}\right) t(t+1)}\left[\frac{a_{3}\left(a_{3}^{t}-a_{2}^{t}\right)}{\left(a_{3}-a_{2}\right)}-\frac{a_{1}\left(a_{2}^{t}-a_{1}^{t}\right)}{\left(a_{2}-a_{1}\right)}\right] .
$$

Thus the mean $\left(\mu_{\widetilde{A}_{\tilde{A}_{1}}}\right)$ and variance $\left(\sigma_{\widetilde{X}_{\tilde{A}_{1}}}^{2}\right)$ of the random variable $X_{\widetilde{A}_{1}}$ can be obtained as

$$
\begin{aligned}
& \mu_{\widetilde{A}_{\tilde{A}_{1}}}=\mathbb{E}\left[X_{\tilde{A}_{1}}\right]=M_{{X_{\tilde{A}_{1}}}}(2)=\frac{a_{1}+a_{2}+a_{3}}{3},
\end{aligned}
$$

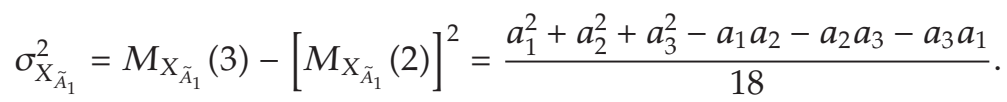

Further, the probability density function corresponding to trapezoidal fuzzy number $\widetilde{A}_{2}=$ $\left(a_{1}, a_{2}, a_{3}, a_{4}\right)$ is given as $f_{\widetilde{A}_{2}}(x)=c_{2} \mu_{\tilde{A}_{2}}(x)$, where $\mu_{\widetilde{A}_{2}}(x)$ is defined as

$$
\mu_{\tilde{A}_{2}}(x)= \begin{cases}\frac{x-a_{1}}{a_{2}-a_{1}}, & a_{1} \leq x \leq a_{2} \\ 1, & a_{2} \leq x \leq a_{3} \\ \frac{a_{4}-x}{a_{4}-a_{3}}, & a_{3} \leq x \leq a_{4} \\ 0, & \text { otherwise. }\end{cases}
$$


Now $c_{2}$ is calculated as

$$
\int_{-\infty}^{\infty} f_{\tilde{A}_{2}}(x) d x=1
$$

that is,

$$
\int_{-\infty}^{\infty} c_{2} \mu_{\tilde{A}_{1}}(x) d x=1
$$

that is,

$$
c_{2} \int_{a_{1}}^{a_{2}} \frac{x-a_{1}}{a_{2}-a_{1}} d x+c_{2} \int_{a_{2}}^{a_{3}} d x+c_{2} \int_{a_{3}}^{a_{4}} \frac{a_{4}-x}{a_{4}-a_{3}} d x=1
$$

On integration, we get

$$
c_{2}=\frac{2}{a_{4}+a_{3}-a_{1}-a_{2}} .
$$

The proportional probability density function corresponding to triangular fuzzy number $\widetilde{A}_{1}$ is given by

$$
f_{X_{\tilde{A}_{2}}}(x)= \begin{cases}\frac{2\left(x-a_{1}\right)}{\left(a_{2}-a_{1}\right)\left(a_{4}+a_{3}-a_{1}-a_{2}\right)}, & a_{1} \leq x \leq a_{2} \\ \frac{2}{\left(a_{4}+a_{3}-a_{1}-a_{2}\right)}, & a_{2} \leq x \leq a_{3} \\ \frac{2\left(a_{4}-x\right)}{\left(a_{4}-a_{3}\right)\left(a_{4}+a_{3}-a_{1}-a_{2}\right)}, & a_{3} \leq x \leq a_{4} \\ 0, & \text { otherwise. }\end{cases}
$$

Graphically it is shown in Figure 2.

Using the Mellin transform, we get

$$
\begin{aligned}
M_{\tilde{A}_{2}}(t)= & \int_{0}^{\infty} x^{t-1} f_{X_{\tilde{A}_{2}}}(x) d x=\int_{a_{1}}^{a_{2}} x^{t-1} \frac{2\left(x-a_{1}\right)}{\left(a_{2}-a_{1}\right)\left(a_{4}+a_{3}-a_{1}-a_{2}\right)} d x \\
& +\int_{a_{2}}^{a_{3}} x^{t-1} \frac{2}{\left(a_{4}+a_{3}-a_{1}-a_{2}\right)} d x+\int_{a_{3}}^{a_{4}} x^{t-1} \frac{2\left(a_{4}-x\right)}{\left(a_{4}-a_{3}\right)\left(a_{4}+a_{3}-a_{1}-a_{2}\right)} d x
\end{aligned}
$$

On integration, we obtain

$$
M_{\tilde{A}_{2}}(t)=\frac{2}{\left(a_{4}+a_{3}-a_{1}-a_{2}\right) t(t+1)}\left[\frac{\left(a_{4}^{t+1}-a_{3}^{t+1}\right)}{\left(a_{4}-a_{3}\right)}-\frac{\left(a_{2}^{t+1}-a_{1}^{t+1}\right)}{\left(a_{2}-a_{1}\right)}\right] .
$$




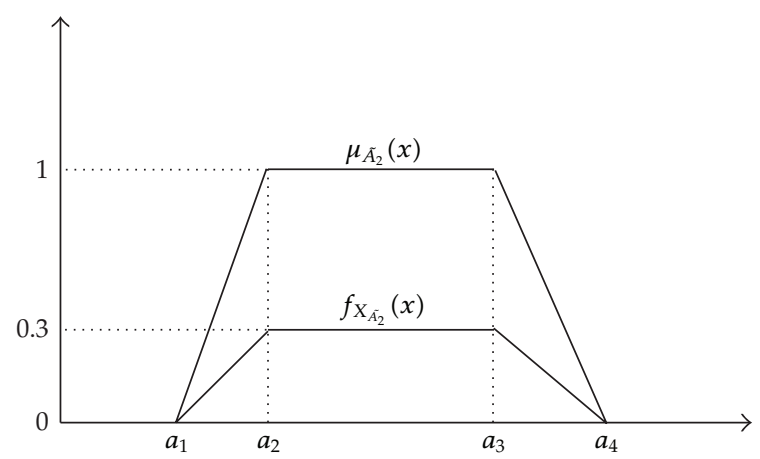

Figure 2: Proportional probability density function of trapezoidal fuzzy number.

Thus, the mean $\left(\mu_{{\widetilde{\tilde{A}_{2}}}_{2}}\right)$ and variance $\left(\sigma_{\widetilde{X}_{\tilde{A}_{2}}}^{2}\right)$ of the random variable $X_{\tilde{A}_{2}}$ can be obtained as

$$
\begin{aligned}
& \mu_{\widetilde{A}_{\tilde{A}_{2}}}=\mathbb{E}\left[X_{\tilde{A}_{2}}\right]=M_{X_{\tilde{A}_{2}}}(2)=\frac{1}{3}\left[\left(a_{1}+a_{2}+a_{3}+a_{4}\right)+\frac{\left(a_{1} a_{2}-a_{3} a_{4}\right)}{\left(a_{4}+a_{3}-a_{2}-a_{1}\right)}\right],
\end{aligned}
$$

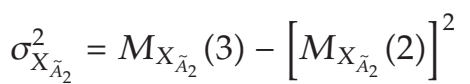

$$
\begin{aligned}
& =\frac{1}{6}\left[\left(a_{1}^{2}+a_{2}^{2}+a_{3}^{2}+a_{4}^{2}\right)+\frac{\left(a_{1}+a_{2}\right)\left(a_{3}^{2}+a_{4}^{2}\right)-\left(a_{3}+a_{4}\right)\left(a_{1}^{2}+a_{2}^{2}\right)}{\left(a_{4}+a_{3}-a_{2}-a_{1}\right)}\right]-\left(\mu_{\tilde{X}_{\tilde{A}_{2}}}\right)^{2} .
\end{aligned}
$$

\subsection{Probabilistic Fuzzy Quadratic Programming Problem and Its Crisp Model}

Let $\tilde{c}_{j}=\left(c_{j}^{1}, c_{j}^{2}, c_{j}^{3}\right), j=1,2, \ldots, n, \tilde{a}_{i j}=\left(a_{i j}^{1}, a_{i j}^{2}, a_{i j}^{3}\right), i=1,2, \ldots, m, j=1,2, \ldots, n$, and $\tilde{q}_{i j}=\left(q_{i j}^{1}, q_{i j}^{2}, q_{i j}^{3}\right), i=1,2, \ldots, n, j=1,2, \ldots, n$ be triangular fuzzy numbers. The crisp values of these fuzzy numbers obtained by using the method of defuzzification with probability density function of given membership function as described in Section 2 are given as

$$
\begin{aligned}
& \widehat{c}_{j}=\frac{c_{j}^{1}+c_{j}^{2}+c_{j}^{3}}{3}, \quad j=1,2, \ldots, n, \\
& \widehat{a}_{i j}=\frac{a_{i j}^{1}+a_{i j}^{2}+a_{i j}^{3}}{3}, \quad i=1,2, \ldots, m, j=1,2, \ldots, n, \\
& \widehat{q}_{i j} \frac{q_{i j}^{1}+q_{i j}^{2}+q_{i j}^{3}}{3}, \quad i=1,2, \ldots, n, j=1,2, \ldots, n,
\end{aligned}
$$

where the symbol $\widehat{c}_{j}$ represents the crisp value of the given fuzzy number $\widetilde{c}_{j}$ and so on. 
Similarly, if all the coefficients are trapezoidal fuzzy numbers such as, $\tilde{c}_{j}=$ $\left(c_{j}^{1}, c_{j}^{2}, c_{j}^{3}, c_{j}^{4}\right), j=1,2, \ldots, n, \tilde{a}_{i j}=\left(a_{i j}^{1}, a_{i j}^{2}, a_{i j}^{3}, a_{i j}^{4}\right), i=1,2, \ldots, m, j=1,2, \ldots, n$, and $\tilde{q}_{i j}=$ $\left(q_{i j}^{1}, q_{i j}^{2}, q_{i j}^{3}, q_{i j}^{4}\right), i=1,2, \ldots, n, j=1,2, \ldots, n$, then the crisp values are given as

$$
\begin{aligned}
& \widehat{c}_{j}=\frac{1}{3}\left[\left(c_{j}^{1}+c_{j}^{2}+c_{j}^{3}+c_{j}^{4}\right)+\frac{\left(c_{j}^{1} c_{j}^{2}-c_{j}^{3} c_{j}^{4}\right)}{\left(c_{j}^{3}+c_{j}^{4}-c_{j}^{1}-c_{j}^{2}\right)}\right], j=1,2, \ldots, n, \\
& \widehat{a}_{i j}=\frac{1}{3}\left[\left(a_{i j}^{1}+a_{i j}^{2}+a_{i j}^{3}+a_{i j}^{4}\right)+\frac{\left(a_{i j}^{1} a_{i j}^{2}-a_{i j}^{3} a_{i j}^{4}\right)}{\left(a_{i j}^{3}+a_{i j}^{4}-a_{i j}^{1}-a_{i j}^{2}\right)}\right], \quad i=1,2, \ldots, m, j=1,2, \ldots, n, \\
& \widehat{q}_{i j}=\frac{1}{3}\left[\left(q_{i j}^{1}+q_{i j}^{2}+q_{i j}^{3}+q_{i j}^{4}\right)+\frac{\left(q_{i j}^{1} q_{i j}^{2}-q_{i j}^{3} q_{i j}^{4}\right)}{\left(q_{i j}^{3}+q_{i j}^{4}-q_{i j}^{1}-q_{i j}^{2}\right)}\right], \quad i=1,2, \ldots, n, j=1,2, \ldots, n .
\end{aligned}
$$

Thus, the probabilistic quadratic programming problem having crisp objective function can be stated as

$$
\min : z=\sum_{j=1}^{n} \widehat{c}_{j} x_{j}+\frac{1}{2} \sum_{i=1}^{n} \sum_{j=1}^{n} \widehat{q}_{i j} x_{i} x_{j}
$$

subject to

$$
\begin{gathered}
\operatorname{Pr}\left(\sum_{j=1}^{n} \widehat{a}_{i j} x_{j} \leq b_{i}\right) \geq 1-\alpha_{i}, \quad i=1,2, \ldots, m, \\
x_{j} \geq 0, \quad j=1,2, \ldots, n,
\end{gathered}
$$

where $0<\alpha_{i}<1, i=1,2, \ldots, m$.

\subsection{Deterministic Model of the Probabilistic Quadratic Programming Problem}

It is assumed that the $i$ th random variables $b_{i}, i=1,2, \ldots, m$ follow the Weibull distribution [14]. The probability density function (pdf) of the random variables $b_{i}$ is given by

$$
f\left(b_{i}\right)= \begin{cases}\left(\frac{p_{i}}{s_{i}}\right)\left(\frac{b_{i}}{s_{i}}\right)^{p_{i}-1} e^{-\left(b_{i} / s_{i}\right)^{p_{i}}} & \text { if } b_{i} \geq 0, p_{i}>0, s_{i}>0 \\ 0, & \text { elsewhere }\end{cases}
$$


with

$$
\begin{gathered}
E\left(b_{i}\right)=s_{i} \Gamma\left(1+\frac{1}{p_{i}}\right), \quad p_{i}>0, s_{i}>0, i=1,2, \ldots, m, \\
\operatorname{Var}\left(b_{i}\right)=s_{i}^{2} \Gamma\left(1+\frac{2}{p_{i}}\right)-\left[s_{i} \Gamma\left(1+\frac{1}{p_{i}}\right)\right]^{2}, \quad p_{i}>0, s_{i}>0, i=1,2, \ldots, m,
\end{gathered}
$$

where $p_{i}, i=1,2, \ldots, m$, and $s_{i}, i=1,2, \ldots, m$, are called shape parameters and scale parameters, respectively.

Now, using pdf of the Weibull distribution, the $i$ th probability constraint can be written as

$$
\int_{y_{i}}^{\infty} f\left(b_{i}\right) d b_{i} \geq 1-\alpha_{i}, \quad i=1,2, \ldots, m
$$

where $y_{i}=\sum_{j=1}^{n} \widehat{a}_{i j} x_{j}$ and $y_{i} \geq 0$.

It can be further written as

$$
\int_{y_{i}}^{\infty}\left(\frac{p_{i}}{s_{i}}\right)\left(\frac{b_{i}}{s_{i}}\right)^{p_{i}-1} e^{-\left(b_{i} / s_{i}\right)^{p_{i}}} d b_{i} \geq 1-\alpha_{i}, \quad i=1,2, \ldots, m,
$$

On integration, we obtain

$$
e^{-\left(y_{i} / s_{i}\right)^{p_{i}}} \geq 1-\alpha_{i}, \quad i=1,2, \ldots, m \text {. }
$$

It can be further simplified as

$$
y_{i} \leq \frac{s_{i}}{\left[\ln \left(1-\alpha_{i}\right)\right]^{1 / p_{i}}}, \quad i=1,2, \ldots, m
$$

that is,

$$
\sum_{j=1}^{n} \widehat{a}_{i j} x_{j} \leq \frac{s_{i}}{\left[\ln \left(1-\alpha_{i}\right)\right]^{1 / p_{i}}}, \quad i=1,2, \ldots, m,
$$

Thus, the equivalent deterministic model of the probabilistic quadratic programming problem (2.28)-(2.30) can be stated as

$$
\min : z=\sum_{j=1}^{n} \widehat{c}_{j} x_{j}+\frac{1}{2} \sum_{i=1}^{n} \sum_{j=1}^{n} \widehat{q}_{i j} x_{i} x_{j}
$$


subject to

$$
\begin{gathered}
\sum_{j=1}^{n} \widehat{a}_{i j} x_{j} \leq \frac{s_{i}}{\left[\ln \left(1-\alpha_{i}\right)\right]^{1 / \mathrm{p}_{i}}}, \quad i=1,2, \ldots, m, \\
x_{j} \geq 0, \quad j=1,2, \ldots, n .
\end{gathered}
$$

\section{Numerical Example}

Let us consider the following probabilistic fuzzy quadratic programming problem:

$$
\min : z=\widetilde{26} x_{1}+\widetilde{35} x_{2}+\frac{1}{2}\left[\widetilde{8} x_{1}^{2}-\widetilde{6} x_{1} x_{2}+\widetilde{8} x_{2}^{2}\right]
$$

subject to

$$
\begin{gathered}
\operatorname{Pr}\left(\widetilde{13} x_{1}+\widetilde{25} x_{2} \leq b_{1}\right) \geq 0.95 \\
\operatorname{Pr}\left(\widetilde{17} x_{1}+\widetilde{23} x_{2} \leq b_{1}\right) \geq 0.85 \\
\widetilde{13} x_{1}+\widetilde{15} x_{2} \geq \widetilde{25} \\
x_{1}, x_{2} \geq 0
\end{gathered}
$$

where $\alpha_{1}=0.05$ and $\alpha_{1}=0.15$ are specified probability levels. The fuzzy coefficients $\widetilde{26}=(24,26,27), \widetilde{35}=(32,35,37)$, and $\widetilde{8}=(5,8,10), \widetilde{6}=(4,6,7), \widetilde{8}=(6,8,11)$ are defined by triangular fuzzy numbers. But the fuzzy coefficients $\widetilde{13}=(10,12,14,17), \widetilde{25}=(22,24,25,27)$, $\widetilde{17}=(14,16,18,22), \widetilde{23}=(21,23,24,26), \widetilde{13}=(10,12,15,17), \widetilde{15}=(12,14,16,20)$, and $\widetilde{25}=$ $(23,25,26,28)$ are defined by trapezoidal fuzzy numbers. The right-hand side parameters $b_{1}$ and $b_{2}$ follow the Weibull distribution with known parameters. The parameters are given as

$$
p_{1}=\frac{1}{2}, \quad p_{2}=\frac{1}{4}, \quad q_{1}=0.12, \quad q_{2}=0.03
$$

The crisp values of the above triangular and trapezoidal fuzzy numbers are calculated by using (2.14) and (2.24). That is, $\widehat{26}=(24+26+27) / 3=25.67, \widehat{35}=(32+35+37) / 3=34.67$, $\widehat{8}=(5+8+10) / 3=7.67, \widehat{6}=(4+6+7) / 3=5.67, \widehat{8}=(6+8+11) / 3=8.33, \widehat{13}=(1 / 3)[(10+12+$ $14+17)+(10 \times 12-14 \times 17) /(14+17-10-12)]=13.36, \widehat{25}=(1 / 3)[(22+24+25+27)+(22 \times$ $24-25 \times 27) /(25+27-22-24)]=24.5, \widehat{17}=(1 / 3)[(14+16+18+22)+(14 \times 16-18 \times 22) /(18+$ $22-14-16)]=17.6, \widehat{23}=(1 / 3)[(21+23+24+26)+(21 \times 23-24 \times 26) /(44+26-21-23)]=$ $23.5, \widehat{13}=(1 / 3)[(10+12+15+17)+(10 \times 12-15 \times 17) /(15+17-10-12)]=13.5$, $\widehat{15}=(1 / 3)[(12+14+17+20)+(12 \times 14-16 \times 20) /(16+20-14-12)]=15.6, \widehat{25}=$ $(1 / 3)[(23+25+26+28)+(23 \times 25-26 \times 28) /(26+28-23-25)]=25.5$.

Now, using the parameter values of the random variables, crisp values of the triangular and trapezoidal fuzzy numbers, and the deterministic constraint (2.38), we formulate an 
equivalent deterministic crisp model of the given probabilistic fuzzy quadratic programming model problem:

$$
\min : z=25.67 x_{1}+34.67 x_{2}+\frac{1}{2}\left[8.33 x_{1}^{2}-5.67 x_{1} x_{2}+7.67 x_{2}^{2}\right]
$$

subject to

$$
\begin{gathered}
13.36 x_{1}+24.5 x_{2} \leq 45.610034, \\
17.6 x_{1}+23.5 x_{2} \leq 42.979943 \\
13.5 x_{1}+15.6 x_{2} \geq 25.5, \\
x_{1}, x_{2} \geq 0 .
\end{gathered}
$$

The above deterministic crisp quadratic programming problem is solved using LINGO 11.0 [15] software. The optimal solution is obtained as $x_{1}=0.9623144, x_{2}=0.9394838$, and $z=$ 62.91564 .

\section{Conclusions}

Quadratic programming is one of the important techniques for improving the efficiency and increasing the productivity of business companies and public organizations. It is also applied to some of the managerial decision making problems such as demand-supply response and enterprise selection. In this paper, a single-objective probabilistic fuzzy quadratic programming problem is presented where both fuzziness and randomness are involved within the quadratic programming framework. The present work can be extended in the multiobjective framework considering the parameters as different random variables and other fuzzy numbers depending on the problems.

\section{References}

[1] B. A. McCarl, H. Moskowitz, and H. Furtan, "Quadratic programming applications," Omega, vol. 5, no. 1, pp. 43-55, 1977.

[2] S. T. Liu and R.-T. Wang, "A numerical solution method to interval quadratic programming," Applied Mathematics and Computation, vol. 189, no. 2, pp. 1274-1281, 2007.

[3] R. C. Silva, J. L. Verdegay, and A. Yamakami, "Two-phase method to solve fuzzy quadratic programming problems," in Proceedings of the IEEE International Conference on Fuzzy Systems: FUZZ-IEEE, pp. 1-6, London, UK, July 2007.

[4] S. T. Liu, "Solving quadratic programming with fuzzy parameters based on extension principle," in Proceedings of the IEEE International Conference on Fuzzy Systems: FUZZ-IEEE, pp. 1-5, London, UK, July 2007.

[5] E. E. Ammar, "On solutions of fuzzy random multiobjective quadratic programming with applications in portfolio problem," Information Sciences, vol. 178, no. 2, pp. 468-484, 2008.

[6] S. T. Liu, "Quadratic programming with fuzzy parameters: a membership function approach," Chaos, Solitons and Fractals, vol. 40, no. 1, pp. 237-245, 2009.

[7] S. T. Liu, "A revisit to quadratic programming with fuzzy parameters," Chaos, Solitons and Fractals, vol. 41, no. 3, pp. 1401-1407, 2009. 
[8] X.S. Qin and G. H. Huang, "An inexact chance-constrained quadratic programming model for stream water quality management," Water Resources Management, vol. 23, no. 4, pp. 661-695, 2009.

[9] S. H. Nasseri, "Quadratic programming with fuzzy numbers: a linear ranking method," Australian Journal of Basic and Applied Sciences, vol. 4, no. 8, pp. 3513-3518, 2010.

[10] P. Guo and G. H. Huang, "Inexact fuzzy-stochastic quadratic programming approach for waste management under multiple uncertainties," Engineering Optimization, vol. 43, no. 5, pp. 525-539, 2011.

[11] A. D. Poularikas, Transforms and Applications Handbook, vol. 43 of The Electrical Engineering Handbook Series, CRC Press, Boca Raton, Fla, USA, 3rd edition, 1996.

[12] R. Saneifard and R. Saneifard, "A modified method for defuzzification by probability density function," Journal of Applied Sciences Research, vol. 7, no. 2, pp. 102-110, 2011.

[13] K. P. Yoon, "A probabilistic approach to rank complex fuzzy numbers," Fuzzy Sets and Systems, vol. 80, no. 2, pp. 167-176, 1996.

[14] N. L. Johnson, S. Kotz, and N. Balakrishnan, Continuous Univariate Distributions - I, John Wiley \& Sons, New York, NY, USA, 2nd edition, 1994.

[15] L. Schrage, Optimization Modeling with LINGO, LINDO Systems, Chicago, Ill, USA, 6th edition, 2006. 


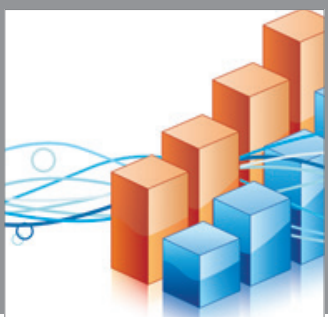

Advances in

Operations Research

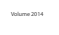

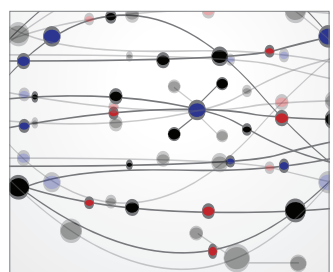

\section{The Scientific} World Journal
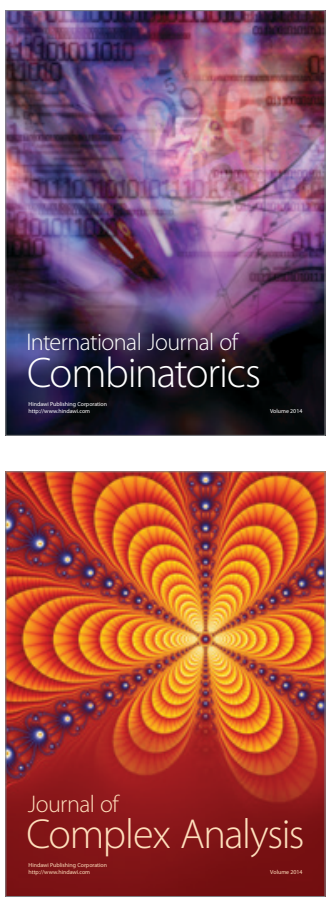

International Journal of

Mathematics and

Mathematical

Sciences
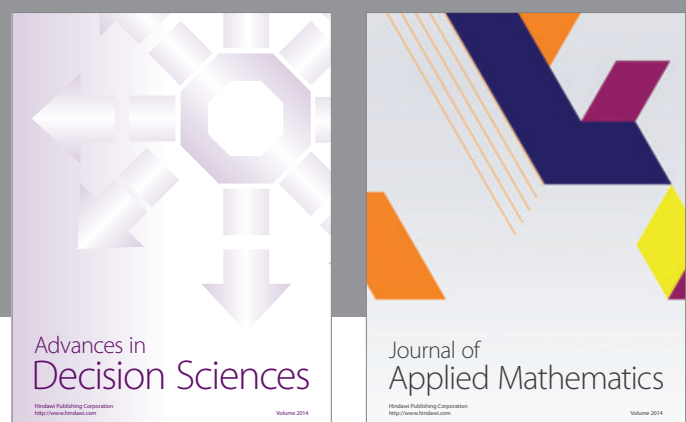

Journal of

Applied Mathematics
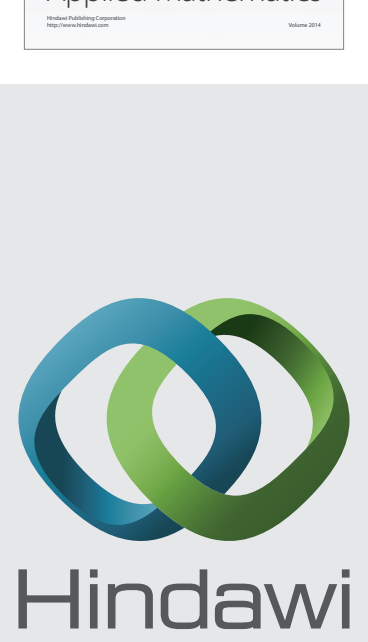

Submit your manuscripts at http://www.hindawi.com
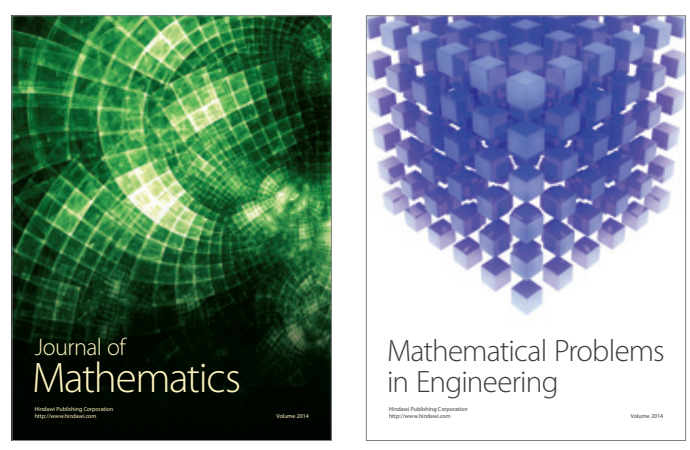

Mathematical Problems in Engineering
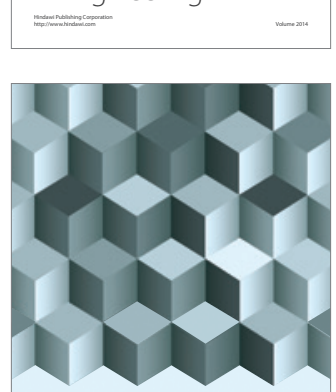

Journal of

Function Spaces
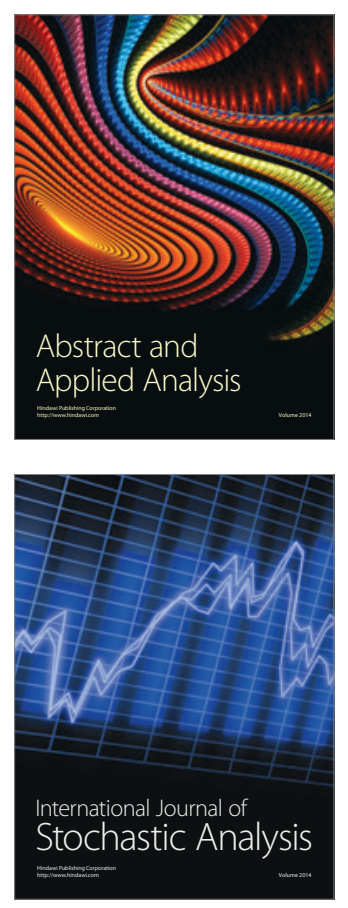

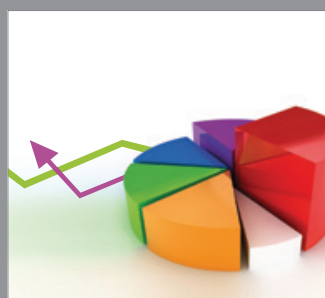

ournal of

Probability and Statistics

Promensencen
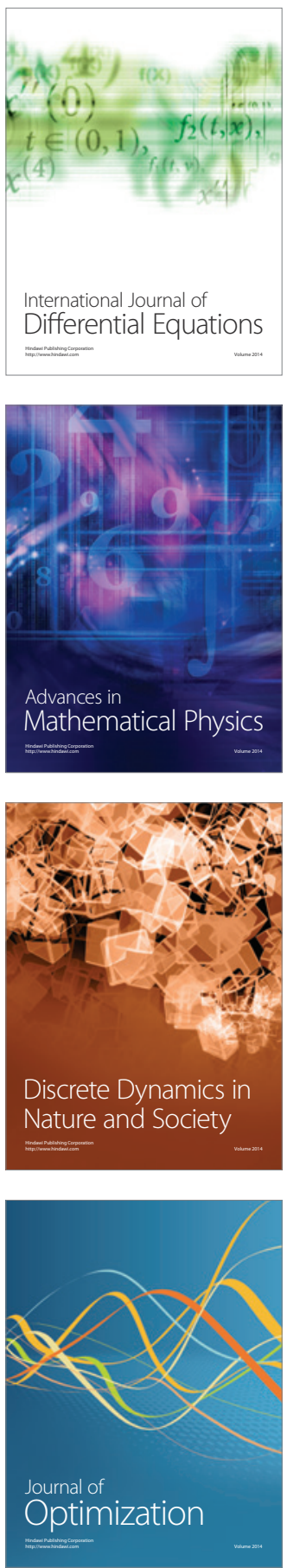